\title{
Suprathreshold binocular interactions for grating patterns
}

\author{
RONALD S. HARWERTH, EARL L. SMITH III, and DENNIS M. LEVI \\ College of Optometry, University of Houston, Houston, Texas 77004
}

\begin{abstract}
Binocular interactions of suprathreshold grating patterns have been investigated using a reaction time measure of contrast detection. Simple reaction times were determined for monocular and binocular viewing conditions over a contrast range from .63 to near threshold. The results from all subjects showed binocular summation for contrast levels near threshold, but there was considerable variation across subjects for contrast levels above threshold. Some subjects showed summation over the entire contrast range, but other subjects showed either binocular inhibition or binocular facilitation for some range of contrast levels. The pattern of binocular interaction for a given subject was consistent for several spatial frequencies. The differences in types of interaction between subjects, the variation in magnitude of binocular interaction with contrast level for each subject, and the data from experiments involving stimulation of noncorresponding retinal areas show that the binocular interactions found for suprathreshold stimuli cannot be accounted for on the basis of probability, and must, therefore, result from physiological interactions between the two eyes. These interactions have been investigated further under conditions of (1) induced fixation disparity, (2) horizontal gratings, and (3) orthogonally oriented gratings.
\end{abstract}

The primary advantages of binocular over monocular vision, for individuals with normal visual acuity in each eye, are stereopsis and binocular summation; however, binocular summation is generally considered to be a rather subtle effect compared to the large advantage of binocular viewing for depth discrimination. Nonetheless, binocular summation has been demonstrated for human observers in a large number of psychophysical experiments (see Blake \& Fox, 1973, for a review). However, in the majority of these studies, threshold measures of stimulus detectability have been used to compare monocular and binocular performance. For example, it has been shown that over a wide range of spatial frequencies, the binocular contrast thresholds for sinusoidal grating patterns are consistently lower than the monocular contrast thresholds (Blake \& Levinson, 1977; Campbell \& Green, 1965a). Specifically, Campbell and Green (1965a) found that the average ratio of binocular to monocular contrast sensitivity was approximately equal to $\sqrt{2}$, a value predicted by assuming summation between two independent channels with uncorrelated noise. They state that since the increased contrast sensitivity is greater than that predicted from probability summation, their results show physiological summation.

We thank Dr. Douglas D. Miller for building and programming the microprocessor used in these experiments. This work was supported by National Eye Institute Grants R01 EY01139, K07 EY00052, F32 EY015168, and R01 EY01768. E. L. S. also received support from a University of Houston Research Initiation Grant.
In contrast to the rather small advantage of binocular over monocular vision found in psychophysical experiments (e.g., approximately 40\%), many electrophysiological experiments have shown substantial effects of binocular stimulation (e.g., greater than $300 \%$ ) (Baker, Grigg, \& von Noorden, 1974; Fischer \& Kruger, 1979; Pettigrew, Nikara, \& Bishop, 1968; Poggio \& Fischer, 1977; von der Heydt, Adorjani, Hanny, \& Baumgartner, 1978). For example, it has been shown with primates that the response of a single cortical neuron may change from binocular facilitation to occlusion as the relative retinal disparity of the stimuli is manipulated. (Baker, Grigg, \& von Noorden, 1974; Poggio \& Fischer, 1977). Additionally, binocular facilitation has been shown in humans for suprathreshold stimuli using the visual evoked response (Apkarian, Nakayama, \& Tyler, 1977; Srebro, 1978). The reason that the magnitude of the binocular interactions, especially binocular facilitation, found in these electrophysiological studies is considerably larger than those found in psychophysical studies may be in part attributable to differences in stimulus levels. The stimuli used in electrophysiological experiments are at suprathreshold levels. Therefore, the relatively small quantifiable improvement in performance for binocular viewing conditions shown in psychophysical experiments may be the result of restricting the stimuli to near threshold levels and it is possible that different types of binocular interactions may be revealed with suprathreshold stimulus levels.

One reason for the lack of psychophysical data for suprathreshold stimuli is that it is difficult to quantify responses for these stimuli. However, simple reaction 
time measurements of grating detection provide a convenient method for quantifying responses for suprathreshold grating stimuli. Harwerth and Levi (1978a) have employed this technique to investigate suprathreshold detection of gratings for monocular viewing and have shown that reaction time increased for all spatial frequencies as the grating contrast was decreased. If it is assumed that simple reaction time is directly correlated to the perceptual strength of the stimulus and that stimuli with equal latencies have equal perceptual values (Harwerth \& Sperling, 1975; Mansfield, 1973; McGill, 1961; Minucci \& Conners, 1964; Roufs, 1974; Stebbins, 1966), ${ }^{1}$ then an isocontrast relationship between monocular and binocular viewing can be derived from reaction time vs. stimulus contrast functions. Thus the relative visibility of a grating pattern for binocular and monocular viewing can be equated by selecting contrast levels which give equal reaction times under the two viewing situations. The use of a series of equal or "criterion" reaction times, therefore, provides a method of determining the magnitude of binocular interaction over a wide range of contrast levels.

An important issue in the investigation of binocular interactions at either threshold or suprathreshold levels is the need to differentiate between effects resulting from probability summation and physiological interactions. While the use of suprathreshold stimulus levels eliminates probability of detection considerations, there are probability models of reaction times that may predict faster mean reaction times for binocular than for monocular viewing (e.g., McGill, 1963). However, both Thorn and Boynton (1974) and Westendorf and Fox (1977) have stated that it is very difficult to obtain the precise conditions necessary to predict probability summation from theoretical models and that it is, therefore, preferable to use totally empirical tests. One strategy that may be employed to determine empirically whether differences in performance between binocular and monocular vision result from physiological neural interactions or simply probabilistic events is to compare data for stimuli on corresponding and noncorresponding retinal areas (e.g., Westendorf \& Fox, 1977). Probability summation would not require the stimulus to be imaged on corresponding areas, but it would be a requirement for physiological interactions. For this reason, reduced binocular interactions when noncorresponding areas rather than corresponding areas are stimulated would indicate the presence of a neural component. In addition to the basic experiments using corresponding and noncorresponding retinal areas, there are several other stimulus conditions that may provide interesting data on the nature of binocular interactions for suprathreshold grating patterns. (1) Physiological studies of primates have shown that the type and magnitude of binocular interaction may be altered by relatively small changes in the retinal disparity of the visual stimuli (Baker, Grigg, \& von Noorden, 1974; Poggio \& Fischer, 1977). These experiments have shown examples in which the response of a single cortical neuron was changed from binocular facilitation to occlusion with a change of retinal disparity as small as 6-12 min of arc. The analogous psychophysical experiment would be one in which binocular interactions were measured with and without an induced fixation disparity. Ogle (1964) has described a method of using vergence eye movements to induce retinal disparities smaller than Panum's fusional areas, thereby producing a fixation disparity while maintaining single binocular vision. Since the stimulus is large with respect to the fixation disparity, similar retinal areas are used in both conditions (except for a small region which is twice the width of the fixation disparity at the edge of the stimulus); therefore, a fixation disparity should not alter probability summation, any change in binocular interaction caused by the introduction of a fixation disparity must represent a neural interaction. (2) It is reasonable to assume that physiological binocular interactions may involve stereodetectors (von Grunau, 1979). In fact, Lema and Blake (1977) have shown that, for threshold stimuli, binocular summation (even probability summation) was absent in stereoblind observers. More recently, Levi, Harwerth, and Manny (1979) have shown that, in humans with amblyopia (and no stereopsis), binocular summation is absent at both threshold and suprathreshold levels and that some amblyopic subjects even show binocular inhibition at suprathreshold levels. Since stereoacuity is optimal for vertical contours (Blake, Camisa, \& Antoinetti, 1976; Ebenholtz \& Walchli, 1965), it might be expected that, for suprathreshold stimuli, normal observers would show greater binocular summation for vertical than for horizontal contours. Although Lema and Blake (1977) have shown equal binocular summation with horizontal and vertical contours at threshold, Apkarian, Nakayama, and Tyler (1977), using the visual evoked response and suprathreshold stimuli, showed that the binocular facilitation present for vertical contours was absent for horizontal contours. Therefore, the investigation of binocular interactions for vertical and horizontal contours may provide some interesting data on the nature of the facilitory interactions at suprathreshold levels. (3) Any binocular interaction of greater magnitude than probability summation requires simultaneous binocular vision (e.g., Battersby \& Defabaugh, 1969; Thorn \& Boynton, 1974). However, when orthogonal contours are presented to corresponding retinal areas of the two eyes, the condi- 
tion of binocular rivalry, rather than simultaneous binocular vision, occurs (Blake \& Fox, 1974). Binocular rivalry is a strong effect phenomenologically, and should be interesting, because the use of rivalry stimuli may provide an opportunity to study binocular interactions in which there may be inhibitory effects between the two eyes.

In aggregate, the stimulus manipulations described should provide empirical data that will make it possible to sort out the contribution of probabilistic events from neural events in the study of binocular interactions for suprathreshold grating stimuli. In the experiments to be reported, binocular interactions for suprathreshold grating stimuli have been investigated using a reaction time paradigm. In the first series of experiments these interactions were studied over a wide range of contrast levels and spatial frequencies in order to identify the range of binocular interactions at suprathreshold levels. In the second series of experiments, binocular interactions were studied under the conditions of (1) noncorresponding retinal images, (2) induced fixation disparity, (3) horizontal gratings, and (4) orthogonal gratings.

\section{METHOD}

Eight subjects with normal binocular vision, 20/20 visual acuity in each eye, and normal stereoacuity (Wirt Stereotest, Titmus Optical Co.) participated in the experiments. Three of the subjects (the authors) participated in all experiments, while the other five subjects participated only in the first experiment.

Sinusoidal gratings were electronically generated on the CRT of an oscilloscope (Tektronix 7603 with a $\mathrm{P} 4$ phosphor), using the method described by Campbell and Green (1965b). The screen was masked to subtend a 4-deg visual angle at the $114-\mathrm{cm}$ viewing distance used in the experiments. The spaceaveraged mean luminance of the screen was $67 \mathrm{~cd} / \mathrm{m}^{2}$ and was constant for all contrast levels and spatial frequencies. The subjects viewed the screen with natural pupils.

A digital process control system (BRS/LVE), interfaced with a microprocessor (SWTP-6800), was used to control the experimental parameters and collect the data. In each trial, following the onset of a ready signal, the subject depressed a response lever to initiate a variable-duration foreperiod. During the foreperiod, the visual stimulus could occur with a .02 probability at the end of any consecutive $100-\mathrm{msec}$ period. The reaction time was measured from the onset of the stimulus to the subject's lever release. The stimulus had square-wave onset and offset properties with a duration of $500 \mathrm{msec}$. If the subject did not release the lever within $1 \mathrm{sec}$ of the onset of stimulus, it was assumed that the grating had not been detected and the reaction time for that trial was excluded from the data analysis; all reaction times shorter than $1 \mathrm{sec}$ were included. Following each reaction time measurement, there was a $4-\mathrm{sec}$ interval before the next trial commenced. Thirty reaction time samples were taken at each contrast level in blocks of 10 trials. The measurements were made at .1-log-unit contrast intervals for each spatial frequency, starting with a maximum of $63 \%$ contrast to a near threshold contrast, i.e., the lowest contrast level at which at least 20 of 30 trials produced reaction time values of less than $1 \mathrm{sec}$.

\section{RESULTS}

Binocular interactions were initially investigated for a $4 \mathrm{c} / \mathrm{deg}$ vertical grating. The $4 \mathrm{c} / \mathrm{deg}$ stimulus was chosen for this experiment because it is at the peak of the contrast sensitivity function and, therefore, provides the largest contrast range over which to investigate binocular interactions. Examples of the mean reaction time as a function of contrast under binocular (open circles) and monocular (filled circles) viewing conditions are shown in Figure 1 for three of the subjects. The subject's dominant eye was always used during the monocular viewing session; however, experiments in which the reaction time data for the two eyes were compared showed that there was no systematic difference between the data for the dominant, i.e., sighting, eye and nondominant eyes. Nor was there any difference in the data for monocular viewing when monocular vision was accomplished by means of a black patch or a diffuser. The standard error of the mean of the reaction times was the smallest for the shortest reaction times and increased for the longer reaction times, but was usually $3 \%-4 \%$ of the mean value for all of the subjects. At the low contrast levels, the mean reaction times were shorter with binocular viewing than with monocular viewing for all of the subjects, but the relationship between the reaction time and viewing condition varied across subjects for higher contrast levels. For example, in Figure 1 it can be seen that for subject E.S. the mean reaction time is shorter under binocular than under monocular viewing conditions for all contrast levels. However, for subject R.R. the mean reaction times for binocular and monocular viewing conditions are approximately

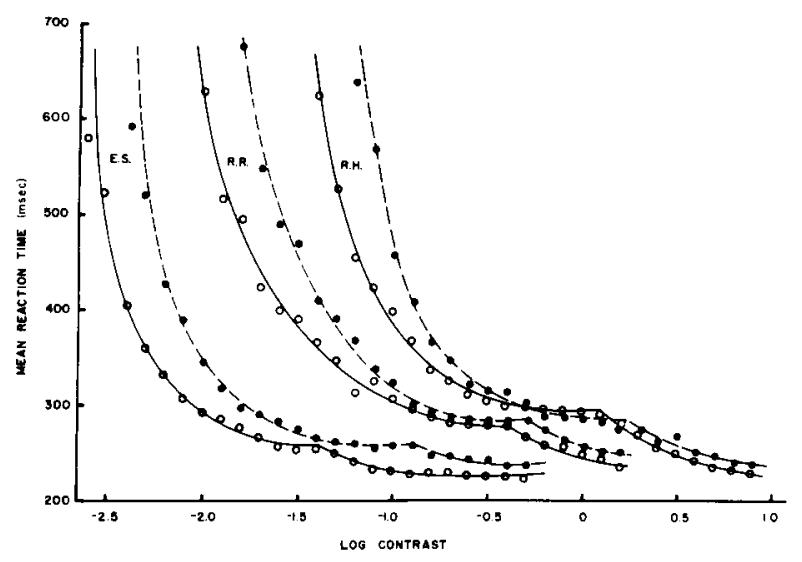

Figure 1. Mean reaction time as a function of contrast for three subjects. The open circles are data for binocular viewing and the filled circles are data for monocular viewing. The curves drawn through the data points were derived from power functions fit to the data. The data for subject E.S. are on a true scale, but the data for R.R. and R.H. have been shifted to the right by .5 and $.6 \log$ units, respectively. 
equal in the midrange contrast levels and for subject R.H. the mean reaction times for these contrast ranges are actually shorter for monocular viewing than for binocular viewing.

The binocular interactions shown by the data in Figure 1 are more clearly displayed by plotting the contrast values for binocular and monocular viewing associated with a series of criterion reaction times. This data transformation was accomplished by curve fitting the mean reaction time data with power functions following the method described by Mansfield (1973). In most cases, the reaction time data were biphasic, as has been reported by Harwerth and Levi (1978a), and, therefore, two separate power functions were fit to the data. The curves drawn through the data in Figure 1 were derived from the power function equations. The correlations coefficients between the curves and the data were .95 or higher for all of the subjects. The binocular interaction plots for a $4 \mathrm{c} / \mathrm{deg}$ stimulus for each of the eight subjects are shown in Figure 2. In these plots, the one-to-one line represents the condition in which equal contrast values result in equal reaction times under either the monocular or the binocular viewing conditions. In order to describe the data efficiently, it is necessary to define adequate descriptive terms. In this context, the terms binocular facilitation, summation, occlusion, and inhibition will be used to characterize the magnitude of binocular performance without meaning to imply explicit physiological mechanisms (Blake \& Fox, 1973). For simplicity, it will be considered that the same improvement in the signal-to-noise ratio would be present at suprathreshold levels that has been documented for threshold measures (Blake \& Levinson, 1977; Campbell \& Green, 1965a). Therefore, binocular summation will be defined as a binocular/ monocular contrast ratio of approximately 1.4 and be represented by a line of unit slope displaced downward by $.15 \mathrm{log}$ unit from the one-to-one line. Binocular facilitation will be defined as binocular/ monocular ratios greater than 1.4, binocular occlusion as ratios between 1.0 and 1.4 , and binocular inhibition as ratios of less than 1.0.

As shown in Figure 2, the binocular/monocular contrast ratios for near-threshold contrast values varied from 1.44 and 1.74 for the eight observers. These values are in adequate agreement with the previous reports of binocular summation for lowcontrast gratings (Blake \& Levinson, 1977; Campbell \& Green, 1965a). However, there is considerable intersubject variability for binocular interaction at suprathreshold contrast levels. Subjects P.P. and R.H. show substantial binocular inhibition over a range of contrast values (the minimum binocular/monocular contrast ratios are .2 and .4 for the two subjects). Three of the subjects, R.D., R.M., and R.R., show small amounts of binocular facilitation that are approximately constant across all contrast levels.

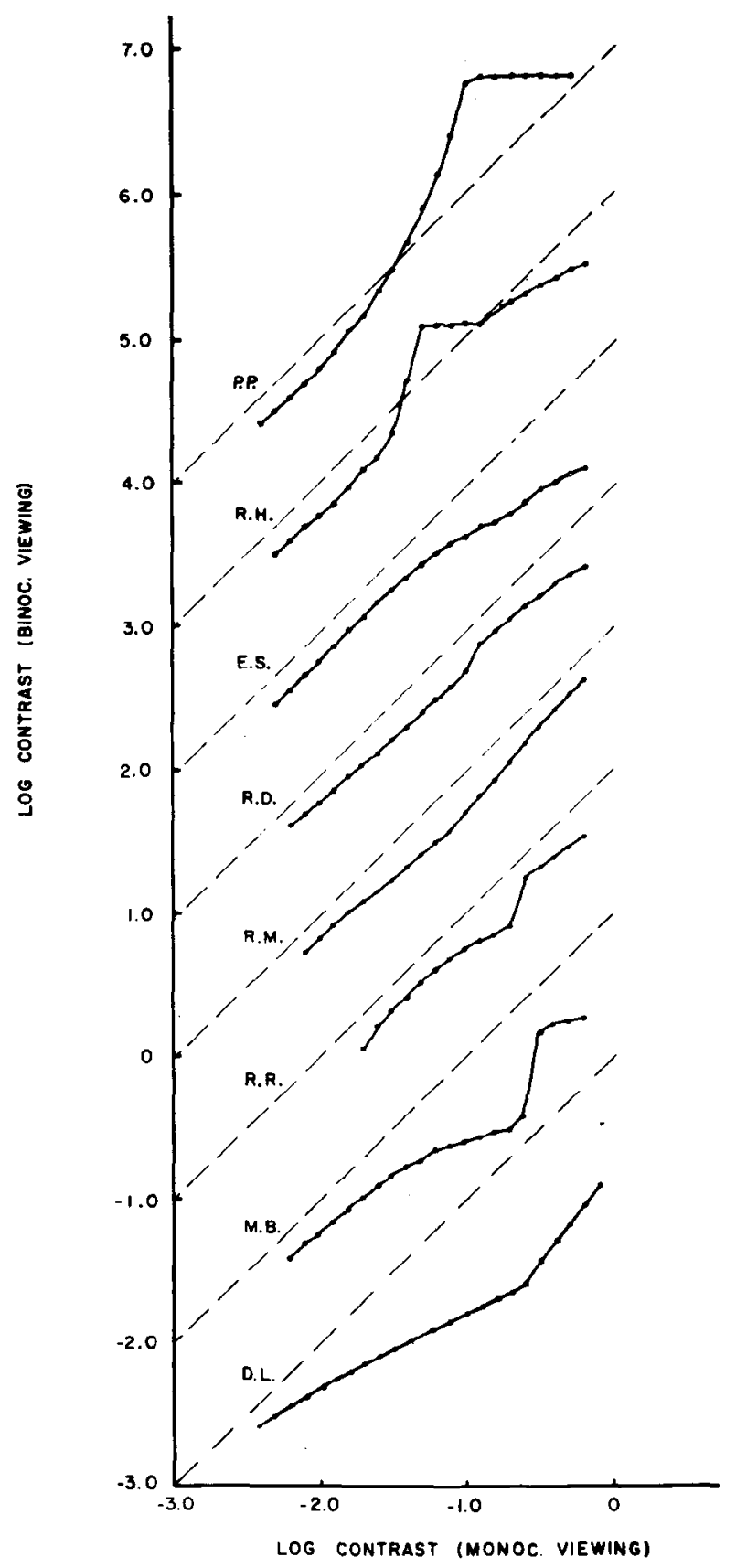

Figure 2. Binocular interaction functions showing the logarithm of the contrast under binocular viewing vs. the logarithm of the contrast under monocular viewing for a series of criterion reaction times. The points were derived from the equations for the power functions fit to the reaction time data (see Figure 1). Points below the line of unit slope represent binocular ocelusion summation or facilitation, and data above the line represent binocular inhibition. Data are shown for eight subjects.

The other three subjects show considerable binocular facilitation for high-contrast stimuli (the maximum binocular/monocular contrast ratios for subjects E.S., M.B., and D.L. are $4.4,6.4$, and 7.8 , respectively), with the magnitude of facilitation decreas- 
ing systematically with decreasing contrast to approximate binocular summation near threshold. All eight of the subjects have normal binocular vision by the usual clinical criteria, and it has not been possible to discover any factor that would predict the binocular interaction patterns of the individual subjects. However, it was discovered after the initial data (shown in Figure 2) had been collected that subject P.P. was a meridional amblyope due to a high degree of astigmatism present early in life (Mitchell, Freeman, Millodot, \& Haegerstrom, 1973). His high-frequency cutoff spatial frequencies with binocular viewing were $32 \mathrm{c} / \mathrm{deg}$ for vertical gratings and $18 \mathrm{c} / \mathrm{deg}$ for horizontal gratings with optimal correction of his astigmatism. The principal meridia of his astigmatism were symmetrical and the vertical orientation of the grating had been optimal for each of the two eyes. Additional data were collected for subject P.P. with a horizontal grating orientation. In Figure 3, the reaction time data and the binocular interaction data are shown for each of these two orientations. The reaction time data show that the reaction times for the two stimulus orientations are not very different for high-contrast levels, but they clearly show the effect of the meridional amblyopia at lower contrast levels. These data are in agreement with those of Georgeson and Sullivan (1975) in showing a considerable suprathreshold contrast compensation for the orientation-specific deficit in meridional amblyopia; however, in strabismic and anisometropic amblyopia, an equivalent suprathreshold compen-

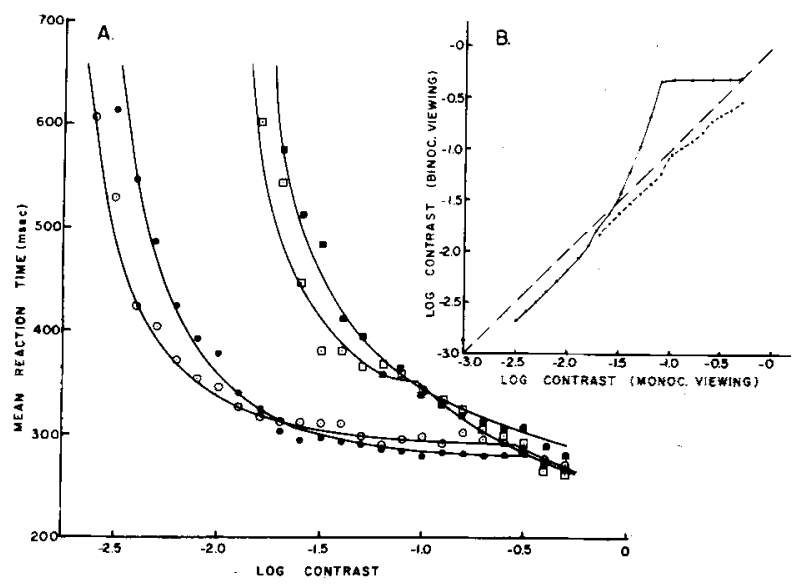

Figure 3. (A) Mean reaction time as a function of contrast for subject P.P., a meridional amblyope. The circles are data for the nonamblyopic meridian, and the squares are the data for the amblyopic meridian, with the open and filled symbols representing data for binocular and monocular viewing, respectively. The curves drawn through the data points are derived from power functions fit to the data. (B) Binocular interaction functions showing the logarithm of the contrast under binocular viewing vs. the logarithm of the contrast under monocular viewing for a series of criterion reaction times. The function for the nonamblyopic meridian is shown by the solid line, and data for the amblyopic meridian are shown by the dashed line.

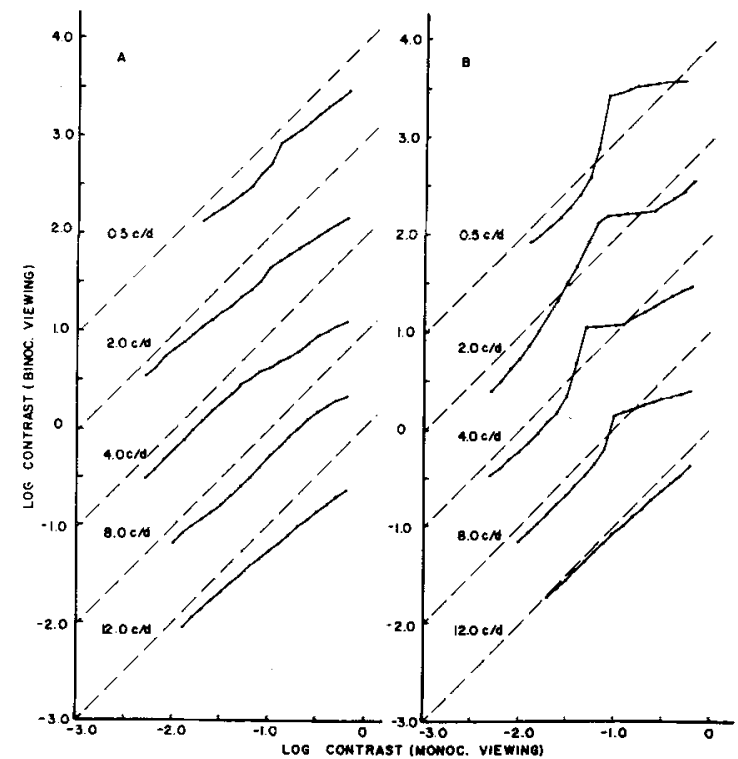

Figure 4. Binocular interaction functions as a function of spatial frequency for two subjects. See Figure 2 for details.

sation does not occur (Harwerth \& Levi, 1978b; Levi, Harwerth, \& Manny, 1979). The binocular interaction data for the meridional amblyope, Figure 3B, also show qualitatively different interactions for the two stimulus orientations. The data for the vertical grating (reproduced from Figure 2) show binocular inhibition for high-contrast levels and approximate summation for the lower contrast values. On the other hand, the binocular interactions for the horizontal grating are nearly equal for all contrast levels and not substantially different from binocular summation. However, as will be seen in experiments to be described, other subjects who are not meridional amblyopes show similar orientation effects. Therefore, these differences in the binocular interactions may represent a characteristic of the orientation of the grating rather than the meridional amblyopia.

Binocular interaction as a function of spatial frequency was investigated for two subjects (E.S. and R.H.) for spatial frequencies of .5, 2.0, 4.0, 8.0, and $12.0 \mathrm{c} / \mathrm{deg}$. The results from this experiment are shown in Figure 4. It can be seen that the patterns of binocular interaction are qualitatively similar across spatial frequencies for each subject. The data for subject E.S. show binocular facilitation for highcontrast stimuli of all spatial frequencies and then a systematic decrease in facilitation toward binocular summation at near threshold. The data for subject R.H. show some amount of binocular inhibition over a similar contrast range for each of the spatial frequencies, except the $12.0 \mathrm{c} / \mathrm{deg}$ stimulus.

Since binocular interaction at suprathreshold levels differs from that observed near threshold, experiments were undertaken to investigate the roles of 
probabilistic events and neural effects in the binocular interactions shown in Figures 2 and 4 . Figure $5 \mathrm{~A}$ shows the data for an experiment in which the diplopic stimuli were imaged on noncorresponding retinal areas. (The stimulus conditions are shown as an inset on Figure 5A.) Fixation points were placed $.12 \mathrm{deg}$ above and below the stimulus area. Monocular viewing data were collected for the right eye with fixation below the stimulus field and for the left eye with fixation above the field. It was found that the data for the two monocular viewing conditions were essentially identical. Since the similarity of the reaction time data for the monocular viewing conditions indicated that the two retinal areas were equivalent in sensitivity, the mean monocular data were used for the comparison to binocular data. Binocular viewing data were collected with (1) binocular fixation below the stimulus area in order to investigate binocular interactions in nonfoveal, corresponding retinal areas, and (2) sufficient vertical prism to separate the retinal images to an extent that the top and bottom fixation points could be binocularly fused and the stimulus fall on completely nonoverlapping retinal areas (thus the observer perceived a single fixation point with a stimulus field directly above and below it). The binocular interaction functions are shown in Figure 5A. The functions for the condition of normal binocular viewing with nonfoveal stimulus areas are shown by the solid line, and the data with noncorresponding images are shown by the dashed line.

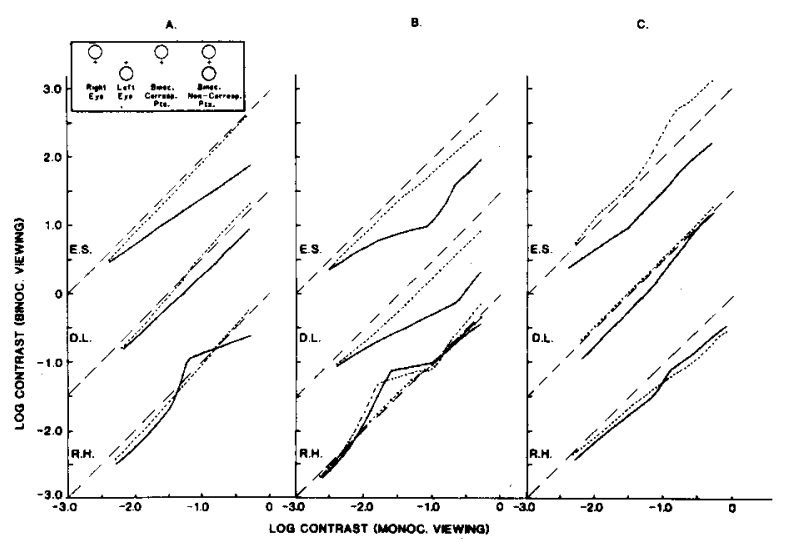

Figure 5. (A) Binocular interaction functions for three subjects for nonfoveal binocular stimuli (solid line) and with gratings on noncorresponding retinal areas (dashed line). The stimulus conditions are illustrated in the upper inset. (B) Binocular interaction functions for three subjects for normal binocular viewing (solid line) and with an induced fixation disparity of $4 \mathrm{~min}$ of arc (dashed line). For subject R.H., data are also shown for an experiment in which he wore 2 prism diopters of base-out and 1 prism diopter of base-up prism before his right eye (dashed line with Xs). (C) Binocular interaction functions for three subjects with horizontal gratings (solid line) and orthogonal gratings (dashed line).
The nature of the binocular interactions for extrafoveal areas are not very different from those shown in Figure 5B with foveal fixation. The binocular interaction functions for the noncorresponding retinal areas are relatively consistent across the three subjects. All three subjects show slight inhibition or zero interaction with high-contrast stimuli and a systematic shift to a binocular/monocular contrast ratio of approximately 1:2 near threshold. Since neural interactions are precluded by spatial separation, the binocular interactions shown in the condition of noncorresponding retinal areas must reflect probabilistic events.

In Figure 5B, the effect of induced fixation disparity is shown for three observers. The fixation disparity was induced by having the subjects view the stimulus with 10 prism diopters of base-in prism for subjects E.S. and R.H. and 8 prism diopters for D.L. With the prisms in place, a fixation disparity of approximately $4 \mathrm{~min}$ of arc $(96 \mathrm{deg}$ phase shift between the two eyes) was measured with orthogonally polarized vernier lines viewed through suitable analyzers while allowing the subjects to maintain binocular fusion. The effects of the fixation disparity can be seen in Figure 5B, in which binocular interactions are shown with normal binocular vision (solid line) and with the induced fixation disparity (dashed line). The data for subjects E.S. and R.H. with normal binocular vision were collected again in this experiment and, therefore, represent a replication of the data shown in Figure 2 . For subjects E.S. and D.L., the data clearly show that the presence of a fixation disparity largely reduces the amount of binocular facilitation for highcontrast levels. The slope of the binocular interaction function with the induced fixation disparity is less than 1.0, indicating some binocular facilitation with high-contrast stimuli, but the magnitude is greatly diminished. For subject R.H., the data with both normal binocular viewing and an induced fixation disparity show binocular inhibition over a similar range of contrasts. In an attempt to determine whether a subtle binocular anomaly might explain the binocular inhibition consistently shown by subject R.H., he underwent a 4-h period of monocular occlusion (Roy, 1969). At the end of the period of monocular occlusion, the prism necessary to completely eliminate his fixation disparity was determined ( 2 prism diopters of base-out and 1 prism diopter of base-up prism before the right eye). The binocular interaction function with these prism lenses in place is shown by the dashed line with $X s$. The effect of this manipulation was to eliminate the binocular inhibition, and the function falls on the one-to-one line over most of the range of contrasts, except at near-threshold values, where it approximates probability summation. 
Figure 5C shows the data from the experiments in which horizontal gratings (solid line) and orthogonal gratings (dashed line) were used to study the binocular interactions. With the horizontal gratings presented to both eyes, the binocular interaction functions show reduced magnitudes of interaction for the high-contrast levels when compared to data obtained with a vertical grating and the same subjects, shown in Figure 5B. Subjects E.S. and D.L. both show reduced facilitation with horizontal gratings, with the data of D.L. showing binocular occlusion for contrasts of greater than $10 \%$. The binocular interaction function for horizontal gratings for subject R.H. shows a small degree of binocular facilitation for suprathreshold contrast levels instead of the binocular inhibition seen with vertical gratings. The data for the functions with the binocular rivalry stimulus are shown by the dashed line in Figure 5C. Gratings with orthogonal orientations were produced via a small Dove prism placed before the left eye of the subject. All three subjects reported that at high stimulus contrast levels the stimulus appeared to be a piecemeal mosaic of the two gratings rather than a domination of one orientation or the other. As the stimulus contrast was reduced, the grating seen by one eye generally became the dominant stimulus in all trials. The data for the three subjects are interesting because they show a different response pattern for each. For subject E.S. the rivalry target causes an apparent binocular inhibition, while subject D.L. shows a binocular response essentially equal to the monocular response and subject R.H. shows binocular facilitation at the highest contrast levels, changing to binocular occlusion at the lower contrast levels.

\section{DISCUSSION}

The results of these experiments show that binocular interactions for suprathreshold grating stimuli are considerably different from those predicted by probability summation. With vertical gratings, all of the subjects showed binocular summation magnitudes for near-threshold stimulus levels that were approximately equal to the value of 1.4 previously reported by other investigators (Blake \& Levinson, 1977; Campbell \& Green, 1965a). The magnitude of binocular summation found in those studies was greater than that predicted from probability summation, and Campbell and Green (1965a) have stated that their results showed physiological summation. In both studies (Blake \& Levinson, 1977; Campbell \& Green, 1965a), the contrast thresholds were determined by the psychophysical method of adjustment using a threshold criterion requiring the grating to be just visible. The method of adjustment applied in such a manner should yield threshold values that would give close to $100 \%$ correct detection performance and, therefore, provide a stronger stimulus than in other studies of binocular summation in which thresholds were determined by the method of constant stimuli. This indication that the magnitude of binocular interaction varies with stimulus strength was also shown in the present experiments using suprathreshold stimuli. However, with the suprathreshold stimuli there were substantial intersubject differences in the type and amount of binocular interaction. The binocular interaction functions for most of the subjects showed a response facilitation when the binocular response was compared to the monocular response, which is in agreement with the report by Minucci and Conners (1964). In contrast, two of the subjects showed a response inhibition for some range of contrast values. The differences in types of interaction between subjects, the variation in magnitude of binocular interaction with contrast level for each subject, and the data from experiments involving (1) noncorresponding retinal areas, (2) induced fixation disparity, and (3) horizontal gratings would indicate that the binocular interactions found for suprathreshold stimuli must result from physiological interactions between the two eyes. Data from the experiment in which stimuli were placed on noncorresponding retinal areas clearly show that the probabilistic events involved in the determination of reaction times for suprathreshold stimuli are not sufficient to explain the improved performance for binocularly viewed stimuli presented to corresponding retinal areas. The data obtained when the gratings were presented to noncorresponding retinal areas show that the improvement from probabilistic events is largest for near-threshold contrast values and systematically decreases with increasing contrast levels, until, at high-contrast levels, probability considerations should not contribute to the binocular interaction. Apkaraian, Nakayama, and Tyler (1977) and Srebro (1978) have shown similar results from visual evoked response experiments. The data for the experiment with induced fixation disparity showed that the presence of a lateral fixation disparity of a smaller amount than the limit of Panum's fusional areas reduced the magnitude of the facilitation for vertical gratings for two of the subjects. However, for these two subjects, the binocular interaction still exceeded that predicted by probability summation, in agreement with the suggestion of Westendorf and Fox (1977) that binocular summation would occur throughout the range of Panum's fusional areas. The finding that the magnitude of binocular interaction is greater for vertical than for horizontal gratings is in agreement with the visual evoked response data of Apkarian, Nakayama, and Tyler (1977), and suggests that stereomechanisms may be 
involved in binocular facilitation. The involvement of stereomechanisms in binocular summation and facilitation has also been suggested by the finding that stereoblind humans (Lema \& Blake, 1977; Levi, Harwerth \& Manny, 1979) and stereoblind cats (von Grunau, 1979) fail to show binocular summation. In the experiments using rivalry grating stimuli, each of the three subjects showed a response pattern for the binocular interaction function which differed from the data with either horizontal or vertical gratings, and also considerable individual variation, which warrants further consideration.

In conclusion, it appears that the reaction time measure of suprathreshold stimulus detection provides a useful method for the investigation of binocular interactions. The experimental data show types and magnitudes of binocular interaction that cannot be accounted for on the basis of probabilistic events, and therefore must represent physiological interactions between the two eyes.

\section{REFERENCES}

Apkarian, P., Nakayama, K., \& Tyler, C. Binocular interactions in steady state visual evoked responses. Society for Neuroscience Abstracts, 1977, 3, 551.

Baker, F. H., Grigg, P., \& von Noorden, G. K. Effects of visual deprivation and strabismus on the response of neurons in the visual cortex of the monkey, including studies on the striate and prestriate cortex in the normal animal. Brain Research, 1974, 66, 185-208.

Battersby, W. S., \& Defabaugh, G. L. Neural limitations on visual excitability: After-effects of subliminal stimulation. Vision Research, 1969, 9, 757-768.

Blake, R., Camisa, J. M., \& Antoinetti, D. N. Binocular depth discrimination depends on orientation. Perception \& Psychophysics, 1976, 20, 113-118.

Blake, R., \& Fox, R. The psychophysical inquiry into binocular summation. Perception \& Psychophysics, 1973, 14, 161-185.

BlAKe, R., \& Fox, R. Binocular rivalry suppression: Insensitive to spatial frequency and orientation change. Vision Research, 1974, 14, 687-692.

Blake, R., \& Levinson, E. Spatial properties of binocular neurons in the human visual system. Experimental Brain Research, 1977, 27, 221-232.

Campbell, F. W., \& Green, D. G. Monocular versus binocular visual acuity. Nature, 1965, 208, 191-192. (a)

Campbell, F. W., \& Green, D. G. Optical and retinal factors affecting visual resolution. Journal of Physiology, 1965, 181, 576-593. (b)

Ebenholtz, S. M., \& Walchli, R. M. Stereoscopic thresholds as a function of head- and object-orientation. Vision Research, $1965,5,455-461$.

Fehrer, E., \& RaAB, D. Reaction time to stimuli masked by metacontrast. Journal of Experimental Psychology, 1962, 63, 143-147.

Fehrer, E., \& Biederman, I. A comparison of reaction time and visual report in the detection of masked stimuli. Journal of Experimental Psychology, 1962, 64, 126-130.

Fischer, B., \& Kruger, J. Disparity tuning and binocularity of single neurons in cat visual cortex. Experimental Brain Research, 1979, 35, 1-8.

Georgeson, M. A., \& Sullivan, G. D. Contrast constancy: Deblurring in human vision by spatial frequency channels. Journal of Physiology, 1975, 252, 627-656.
Harwerth, R. S., \& Levi, D. M. Reaction time as a measure of suprathreshold grating detection. Vision Research, 1978, 18, 1579-1586. (a)

Harwerth, R. S., \& LEvi, D. M. A sensory mechanism for amblyopia: Psychophysical studies. American Journal of Optometry and Physiological Optics, 1978, 55, 151-162. (b)

Harwerth, R. S., \& Sperling, H. G. Effects of intense visible radiation on the increment-threshold spectral sensitivity of the rhesus monkey eye. Vision Research, 1975, 15, 1193-1204.

LEMA, S. A., \& Blake, R. Binocular summation in normal and stereoblind observers. Vision Research, 1977, 17, 691-696.

LEVI, D. M., HARWERTh, R. S., \& MANNY, R. E. Suprathreshold spatial frequency detection and binocular interaction in strabismic and anisometropic amblyopia. Investigative Ophthalmology and Visual Science, 1979, 18, 714-730.

Mansfield, R. J. W. Latency functions in human vision. Vision Research, 1973, 13, 2219-2234.

McGill, W. G. Loudness and reaction time. Psychologica, 1961, 19, 193-199.

McGiLL, W. G. Stochastic latency mechanisms. In R. D. Luce (Ed.), Handbook of mathematical psychology (Vol. 1). New York: Wiley, 1963.

Minucci, P. K., \& Connors, M. M. Reaction time under three viewing conditions: Binocular, dominant eye, and nondominant eye. Journal of Experimental Psychology, 1964, 67, 268-275.

Mitchell, D. E., Freeman, R. D., Millodot, M., \& HaEgerstrom, G. Meridional amblyopia: Evidence for modification of the human system by early visual experience. Vision Research, 1973, 13, 535-558.

Ogle, K. N. Researchers in binocular vision. New York: Hafner, 1964.

Pettigrew, J. D., Nikara, T., \& Bishop, P. O. Binocular interaction on single units in cat striate cortex: Simultaneous stimulation by single moving slit with receptive fields in correspondence. Experimental Brain Research, 1968, 6, 391-410.

Poggio, G. F., \& Fischer, B. Binocular interaction and depth sensitivity in striate and prestriate cortex of behaving rhesus monkey. Journal of Neurophysiology, 1977, 40, 1392-1405.

Rours, J. A. J. Dynamic properties of vision-V. Perceptual lag and reaction time in relation to flicker and flash thresholds. Vision Research, 1974, 14, 853-869.

Roy, R. R. A standardized technique for the prolonged occlusion test. American Journal of Optometry and Archives of the American Academy of Optometry, 1969, 46, 450-456.

Stebrins, W. C. Auditory reaction time and the derivation of equal loudness contours for the monkey. Journal of the Experimental Analysis of Behavior, 1966, 9, 135-142.

Srebro, R. The visually evoked response. Binocular facilitation and failure when binocular vision is disturbed. Archives of Ophthalmology, 1978, 96, 939-944.

Thorn, F., \& Boynton, R. M. Human binocular summation at absolute threshold. Vision Research, 1974, 14, 445-458.

von der Heydt, R., Adorjani, C., Hanny, P., \& Baumgartner G., Disparity sensitivity and receptive field incongruity of units in the cat striate cortex. Experimental Brain Research, 1978, 31, 523-545.

von Grunau, M. Binocular summation and the binocularity of cat visual cortex. Vision Research, 1979, 19, 813-816.

WESTENDORF, D. H., \& Fox, R. Binocular detection of disparate light flashes, Vision Research, 1977, 17, 697-702.

\section{NOTE}

1. An exception to this generally held assumption may occur in masking experiments (Fehrer \& Biederman, 1962; Fehrer \& Raab, 1962); however, the exception should not be a factor in the present study.

(Received for publication February 27, 1979; revision accepted September 28, 1979.) 